\title{
WW PRODUCTION, POLARISATION AND SPIN CORRELATIONS AT LEP
}

\author{
P. AZZURRI \\ Scuola Normale Superiore, Piazza dei Cavalieri 7, 56126 Pisa, Italy \\ E-mail: paolo.azzurri@sns.it
}

\begin{abstract}
Results on W-pair productions at LEP2 are reviewed and their consistency with standard electroweak expectations is summarised. Special interest is given to measurements of W polarisation, and to new studies of spin and decay-plane correlations among the $\mathrm{W}$ pairs.
\end{abstract}

\section{Introduction}

During the data taking years from 1996 to 2000 the LEP2 program has delivered $\mathrm{e}^{+} \mathrm{e}^{-}$ interactions at centre of mass energies ranging from 161 to $207 \mathrm{GeV}$. In this period the four LEP experiments (ALEPH, DELPHI, L3, OPAL) have collected a total integrated luminosity of almost $3 \mathrm{fb}^{-1}$. The LEP2 data yielded about 40,000 $\mathrm{W}$ pair events, reconstructed in all their decay channels. Event rates allowed measurements of the $\mathrm{W}$ pair partial and total cross sections at different energies, as well as of $\mathrm{W}$ decay branching fractions. Further, the study of the W pair decay products kinematics allowed determinations of the $\mathrm{W}$ polarisation states, and of correlations among the two $\mathrm{W}$ bosons.

\section{2. $\mathrm{W}$ pair production rates}

Depending on the decay channels, different techniques allowed to select $\mathrm{W}$ pairs with efficiencies in the 50-90\% range and background contamination in the $5-20 \%$ range $1,2,3,4$. The combined results for the total $\mathrm{W}$ pair cross section at LEP2 energies are shown in figure 1 where the combined experimental precision is at the level of $1 \%$. The total cross section results represent the first clear proof of the electroweak $\mathrm{SU}(2) \otimes \mathrm{U}(1)$ gauge structure, through the gauge cancellations that ensure the $\mathrm{W}$ pair tree level scattering unitarity and the theory renomalizability.

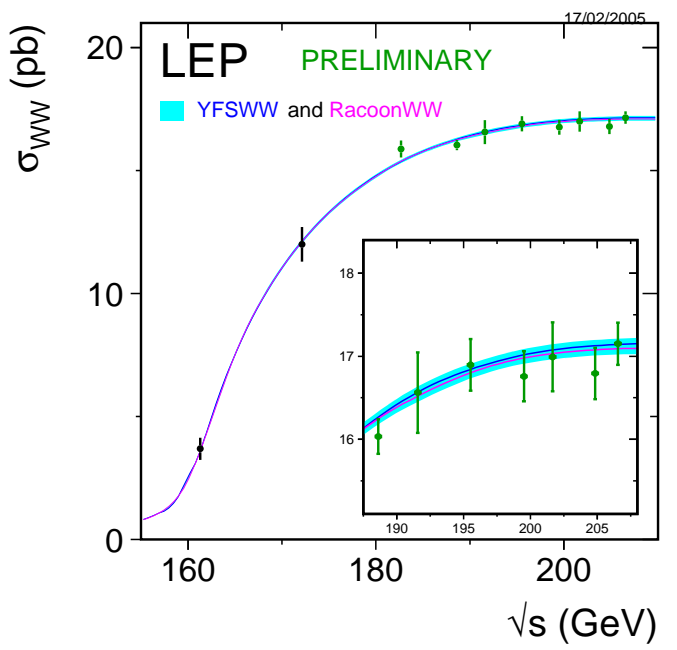

Fig. 1. Total $\mathrm{W}$ pair cross section in $\mathrm{e}^{+} \mathrm{e}^{-}$collisions measured at LEP2 at centre of mass energies from 161 to $207 \mathrm{GeV}$. Dots with error bars are the experimental measurements while the continuos band represents the theoretical predictions based on the Standard Model.

The cross section measurement near the $\mathrm{W}$ pair production kinematic threshold at $\sqrt{s}=161 \mathrm{GeV}$, has allowed a determination of the $\mathrm{W}$ boson mass, $m_{\mathrm{W}}=80.40 \pm$ $0.21 \mathrm{GeV} / \mathrm{c}^{2}$, that is completely independent from the methods based on the kinematics of the decay products. The overall experimental precision of the cross section measurements has also allowed to establish the presence of Standard Model radiative corrections 
to the tree level CC03 diagrams involved in the $\mathrm{W}$ pair production. Depending on the energy, these radiative corrections decrease the total cross section at the $2-3 \%$ level, and modify the shapes of some differential cross section distributions.

The $\mathrm{W}$ pair data has also allowed measurements of the $\mathrm{W}$ decay branching fractions at the $1 \%$ level. Combined results ${ }^{1}$ are in good agreement with the Standard Model expectations, except for the tau leptonic branching fraction that appears to exceed both the electron and muon fractions by more than two standard deviations leading to $2 g_{\tau} /\left(g_{\mathrm{e}}+g_{\mu}\right)=1.036 \pm 0.014$, an overall excess of 2.6 standard deviations.

\section{W Polarisation}

The helicity of the produced W can be measured using the polar and azimuthal decay angles $\left(\theta^{*}, \phi^{*}\right)$ of the decay fermion in the $\mathrm{W}$ rest frame. In the case of a leptonic decay, where the charge of the fermion can be determined, the $\mathrm{W}$ boson fractions of helicity states are given by

$$
\begin{gathered}
\frac{1}{N} \frac{d N}{d \cos \theta_{\ell}^{*}}=f_{-} \frac{3}{8}\left(1+\cos \theta_{\ell}^{*}\right)^{2}+ \\
f_{+} \frac{3}{8}\left(1-\cos \theta_{\ell}^{*}\right)^{2}+f_{0} \frac{3}{4} \sin ^{2} \theta_{\ell}^{*},
\end{gathered}
$$

where $f_{-}, f_{0}, f_{+}$are the fractions of the $\lambda=-1,0,+1$ helicity states for a $\mathrm{W}^{-}$, and of the $\lambda=+1,0,-1$ helicity states for a $\mathrm{W}^{+}$. In the case of hadronic decays, since the charge can't be determined clearly, only the absolute value $\left|\cos \theta_{\mathrm{q}}^{*}\right|$ is measurable and the differential distribution becomes

$$
\begin{array}{r}
\frac{1}{N} \frac{d N}{d\left|\cos \theta_{\mathrm{q}}^{*}\right|}=f_{ \pm} \frac{3}{4}\left(1+\left|\cos \theta_{\mathrm{q}}^{*}\right|^{2}\right)+ \\
f_{0} \frac{3}{4}\left(1-\left|\cos \theta_{\mathrm{q}}^{*}\right|^{2}\right),
\end{array}
$$

where $f_{ \pm}=f_{+}+f_{-}$is the sum of both transverse helicity fractions.

In the case of LEP pair productions, the helicity state will be a function of the centre of mass energy $s$ and of the angle $\theta_{\mathrm{W}^{-}}$of the $\mathrm{W}^{-}$direction with respect to the $\mathrm{e}^{+} \mathrm{e}^{-}$ axis. To measure the helicity fractions, the interference terms and possible $\mathrm{CP}$ or $\mathrm{CPT}$ violating effects, the formalism of the Spin Density Matrix (SDM) is used, defined as

$\sigma_{\tau \tau^{\prime}}^{\mathrm{W}^{-}}\left(s, \cos \theta_{\mathrm{W}^{-}}\right)=F_{\tau}^{\mathrm{W}^{-}}\left(F_{\tau^{\prime}}^{\mathrm{W}^{-}}\right)^{*} / \sum_{\tau}\left|F_{\tau}^{\mathrm{W}^{-}}\right|^{2}$

where $F_{\tau}^{\mathrm{W}^{-}}$is the amplitude to produce a $\mathrm{W}^{-}$with helicity $\tau=-1,0,+1$. The SDM is a Hermitian matrix with unit trace, the diagonal elements are the probabilities to observe a $\mathrm{W}$ in one of the three helicity states and the off diagonal terms represent the interference between helicity amplitudes.

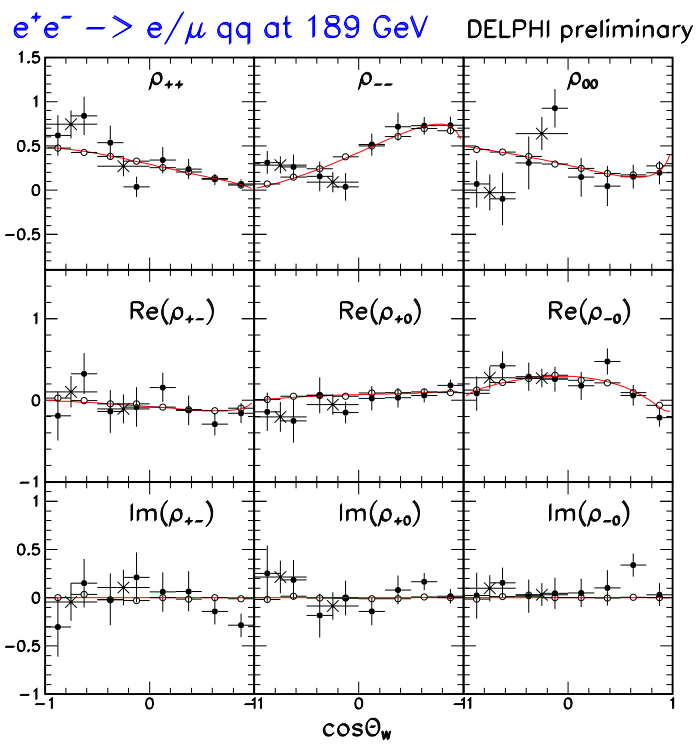

Fig. 2. Measurements of the Spin Density Matrix elements from DELPHI ${ }^{7}$. The values are shown as a function of $\cos \theta_{\mathrm{W}^{-}}$averaging $\mathrm{W}^{-}$and $\mathrm{W}^{+}$data at $\sqrt{s}=189 \mathrm{GeV}$. Full lines and open circles represent Standard Model prediction calculations, while the black dots and crosses are the data values.

LEP experiments have measured SDM elements at different energies and in different $\cos \theta_{\mathrm{W}}$ bins ${ }^{5,6,7}$, by measuring the average values of projection operators $\Lambda_{\tau \tau^{\prime}}^{\mathrm{W}^{-}}$that are analytical functions of the $\theta^{*}$ and $\phi^{*}$ angles. An example of SDM measurements is shown 
in figure 2.

Invariance under $\mathrm{CPT}$ would ensure that $\sigma_{\tau \tau^{\prime}}^{\mathrm{W}^{-}}=\left(\sigma_{-\tau-\tau^{\prime}}^{\mathrm{W}^{+}}\right)^{*}$ while additional CP invariance implies $\sigma_{\tau \tau^{\prime}}^{\mathrm{W}^{-}}=\sigma_{-\tau-\tau^{\prime}}^{\mathrm{W}^{+}}$so that the matrix would be real. Therefore effects that do not conserve $\mathrm{CP}$ or $\mathrm{CPT}$ have been searched for by comparing the $\mathrm{W}^{+}$and $\mathrm{W}^{-}$ SMD elements, and by searching for off diagonal imaginary parts. No significant deviation from the Standard Model expectation has been seen in the data so that both the $\mathrm{CP}$ and $\mathrm{CPT}$ violating effects in the SDM should be at a level smaller that $10^{-1}$.

Focusing on the diagonal SDM elements, the overall fraction of longitudinally polarised W bosons at LEP2 can be extracted averaging $\sigma_{00}$ over all data samples. The average of current results ${ }^{5,6,7}$ yields

$$
f_{L}=\frac{\sigma_{00}}{\sigma_{\mathrm{tot}}}=23.6 \pm 1.6 \% \text {. }
$$

\section{Spin and Decay-Plane Correlations}

Recent studies address the possibility to measure correlations between the spin states of the two $\mathrm{W}$ bosons in $\mathrm{W}$ pair events, and more in general correlations in the decay kinematics of the two $\mathrm{W}$ bosons ${ }^{8}$.

The electroweak model predicts that the helicity combinations of the two $\mathrm{W}$ bosons depend strongly on the direction of the production axis $\cos \theta_{\mathrm{W}^{-}}$as shown in figure 3 . The forward $\cos \theta_{\mathrm{W}^{-}}$direction is where the maximum of $\mathrm{W}$ pairs, both with transverse helicity $\left(\lambda_{\mathrm{W}^{-}}=-1, \lambda_{\mathrm{W}^{+}}=+1\right)$ is found, and selecting $0.3<\cos \theta_{\mathrm{W}^{-}}<0.9,68 \%$ of the $\mathrm{W}$ pairs are expected to be produced in the $(-,+)$ state. Conversely in the backward $\cos \theta_{\mathrm{W}-}$ direction the maximum of $\mathrm{W}$ pairs, with double longitudinal helicity $\left(\lambda_{\mathrm{W}^{-}}=\right.$ $\left.0, \lambda_{\mathrm{W}^{+}}=0\right)$ is expected, in particular a fraction of $28 \%$ in the $-0.9<\cos \theta_{\mathrm{W}^{-}}<-0.3$ region.

Spin correlations have been searched for in semileptonic $\mathrm{W}^{+} \mathrm{W}^{-} \rightarrow \mathrm{q} \overline{\mathrm{q}} \ell \nu$ decays. For

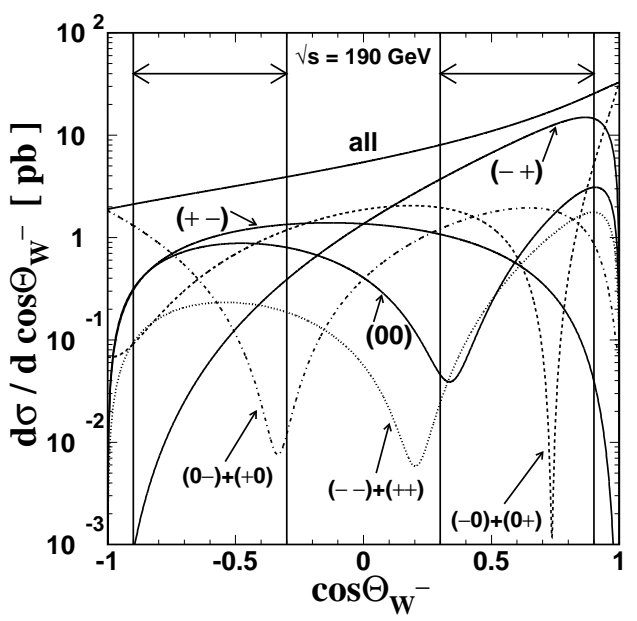

Fig. 3. Differential $\cos \theta_{\mathrm{W}^{-}}$cross section distribution for production of polarised $W$ pairs at $\sqrt{s}=190 \mathrm{GeV}$, from ref. ${ }^{8}$. The figure shows the contribution to the total cross section by each possible pair of $\mathrm{W}^{-}$and $\mathrm{W}^{+}$helicities, indicated in parenthesis. The two $\cos \theta_{\mathrm{W}}$ - intervals indicated by the arrows show the backward region (low $\left.\cos \theta_{\mathrm{W}^{-}}\right)$with increased contributions from $(0,0)$ longitudinal helicities and the forward region (high $\left.\cos \theta_{\mathrm{W}^{-}}\right)$with increased contributions from $(-,+)$ transverse helicities.

the $\mathrm{W}$ decaying hadronically a cut $\left|\cos \theta_{\mathrm{q}}^{*}\right|>$ 0.66 on the polar decay angle distribution allows to select a sample enriched in $\lambda= \pm 1$ transverse spin while a cut $\left|\cos \theta_{\mathrm{q}}^{*}\right|<0.33$ selects a sample of hadronic decays enriched in longitudinal $\lambda=0$ spin. For both samples a fit to the lepton $\cos \theta_{\ell}^{*}$ distribution determines the helicity fractions of the leptonic $\mathrm{W}$, and differences in the fitted fractions can reveal the correlations among the two spin states.

In the forward $\cos \theta_{\mathrm{W}}$ - region, enriching the hadronic transverse spin states should enhance the leptonic transverse spin fractions, while in the backward $\cos \theta_{\mathrm{W}^{-}}$region the enrichment of hadronic longitudinal states should enhance the leptonic longitudinal spin fraction. Data results are shown in figure 4 where the expected effects are visible in the forward $\cos \theta_{\mathrm{W}^{-}}$data, which benefits from quite larger statistics.

With the forward $\cos \theta_{\mathrm{W}^{-}}$data, enrich- 

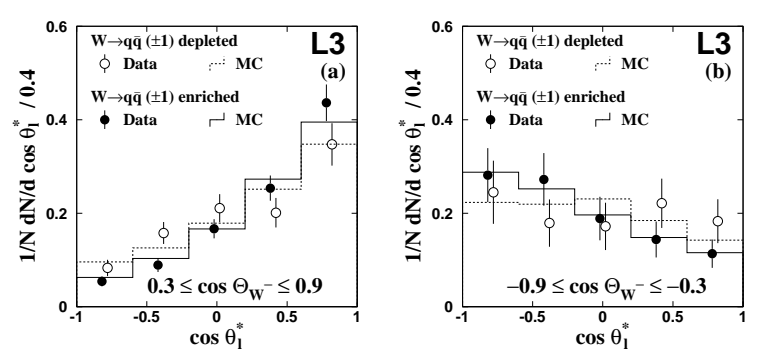

Fig. 4. Distribution of $\cos \theta_{\ell}^{*}$ from L3 ${ }^{8} \mathrm{~W} \rightarrow \ell \nu$ decays, given in the intervals $0.3<\cos \theta_{\mathrm{W}^{-}}<0.9$ (a) and $-0.9<\cos \theta_{\mathrm{W}^{-}}<-0.3(\mathrm{~b})$. Data points and Monte Carlo predictions are shown for two samples, enriched and depleted in transverse helicity for the other $\mathrm{W}$ boson in the event.

ing the hadronic $\mathrm{W}$ transverse spin, with the two $\left|\cos \theta_{\mathrm{q}}^{*}\right|$ selections, changes the leptonic $\mathrm{W}$ helicity fractions by $\Delta f_{-}=+0.32 \pm$ $0.10 \pm 0.06, \Delta f_{+}=-0.03 \pm 0.07 \pm 0.05$ and $\Delta f_{0}=-0.28 \pm 0.14 \pm 0.08$, clearly enhancing the transverse helicities, in reasonable agreement with the Standard Model expectations.
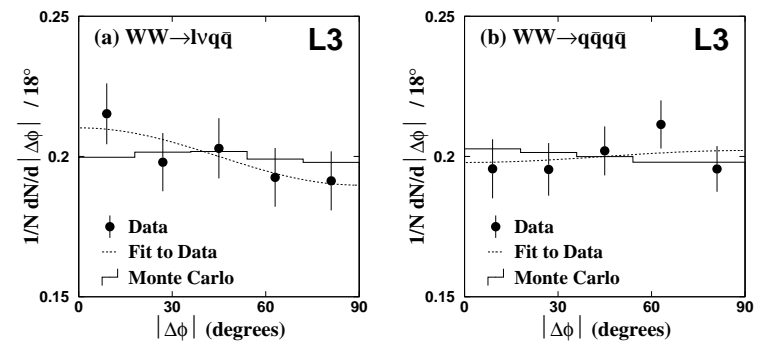

Fig. 5. Distribution of the angle $|\Delta \phi|$ between the decay planes of the two $\mathrm{W}$ bosons from (a) $\mathrm{W}^{+} \mathrm{W}^{-} \rightarrow \ell \nu \mathrm{q} \overline{\mathrm{q}}$ events and (b) $\mathrm{W}^{+} \mathrm{W}^{-} \rightarrow \mathrm{q} \overline{\mathrm{q}} \overline{\mathrm{q}}$ events from L3 ${ }^{8}$. The Monte Carlo predictions are shown with the data points and the results of the fit to the data.

In a more general approach both semileptonic and fully hadronic $\mathrm{W}$ pair decays have been used to determine possible correlations between the the two $\mathrm{W}$ bosons decay planes. Given the angle $|\Delta \phi|$ between the planes of the decay products of the two $\mathrm{W}$ bosons, the differential distribution of $|\Delta \phi|$ is given by

$$
\frac{1}{N} \frac{d N}{d|\Delta \phi|}=1+D \cos |\Delta \phi|
$$

where $D$ measures the strength of the correlation. The data distributions of $|\Delta \phi|$ are shown in figure 5 . The combined result of the fits of the two distributions gives $D=$ $0.012 \pm 0.021 \pm 0.012$, in agreement with the Standard Model Monte Carlo prediction of $D_{M C}=0.010 \pm 0.002$, but not revealing any significant correlation effect.

\section{Conclusions}

The LEP2 data has brought the first precision confirmation of the $\mathrm{SU}(2) \otimes \mathrm{U}(1)$ electroweak gauge structure through the $\mathrm{W}$ pair productions rates and decay kinematics. Results are in good agreement with the Standard Model expectations, with the $\mathrm{W} \tau \nu_{\tau}$ coupling 2.6 standard deviations larger than expected. The $\mathrm{W}$ polarization fractions have been measured accurately and no anomalous interference terms, $\mathrm{CP}$ or $\mathrm{CPT}$ violating contributions have been found in the Spin Density matrix determinations. Spin correlations have also been established between the two $\mathrm{W}$ bosons, in agreement with expectations.

\section{References}

1. The LEP Collaborations, A Combination of Preliminary Electroweak Measurements and Constraints on the Standard Model, CERNPH-EP/2005-051; hep-ex/0511027.

2. The ALEPH Collaboration, Eur.Phys.J. C38, 147 (2004)

3. The DELPHI Collaboration, Eur.Phys.J. C34, 127 (2004)

4. The L3 Collaboration, Phys.Lett. B600, 22 (2004)

5. The L3 Collaboration, Phys.Lett. B557, 147 (2003)

6. The OPAL Collaboration, Phys.Lett. B585, 223 (2004)

7. The DELPHI Collaboration, Contribution to ICHEP04, DELPHI 2004-018 CONF 693.

8. The L3 Collaboration, Eur.Phys.J. C40, 333 (2005) 